\title{
Valence-state reflectometry of complex oxide heterointerfaces
}

\author{
Jorge E Hamann-Borrero ${ }^{1,2}$, Sebastian Macke ${ }^{2,3}$, Woo Seok Choi ${ }^{4,5}$, Ronny Sutarto ${ }^{6}$, Feizhou He ${ }^{6}$, Abdullah Radi ${ }^{2,7}$, llya Elfimov ${ }^{2}$, \\ Robert J Green ${ }^{2,8}$, Maurits W Haverkort ${ }^{8}$, Volodymyr B Zabolotnyy ${ }^{9}$, Ho Nyung Lee ${ }^{4}$, George A Sawatzky ${ }^{2}$ and Vladimir Hinkov ${ }^{2,9}$
}

Emergent phenomena in transition-metal-oxide heterostructures such as interface superconductivity and magnetism have been attributed to electronic reconstruction, which, however, is difficult to detect and characterise. Here we overcome the associated difficulties to simultaneously address the electronic degrees of freedom and distinguish interface from bulk effects by implementing a novel approach to resonant X-ray reflectivity (RXR). Our RXR study of the chemical and valance profiles along the polar (001) direction of $\mathrm{LaCoO}_{3}$ film on $\mathrm{NdGaO}_{3}$ reveals a pronounced valence-state reconstruction from $\mathrm{Co}^{3+}$ in the bulk to $\mathrm{Co}^{2+}$ at the surface, with an areal density close to $0.5 \mathrm{Co}^{2+}$ ions per unit cell. An identical film capped with polar (001) $\mathrm{LaAlO}_{3}$ maintains the $\mathrm{Co}^{3+}$ valence over its entire thickness. We interpret this as evidence for electronic reconstruction in the uncapped film, involving the transfer of $0.5 e^{-}$per unit cell to the subsurface $\mathrm{CoO}_{2}$ layer at its LaO-terminated polar surface.

npj Quantum Materials (2016) 1, 16013; doi:10.1038/npjquantmats.2016.13; published online 16 September 2016

\section{INTRODUCTION}

Heterostructures comprising transition-metal oxides (TMOs) exhibit a particularly rich variety of phenomena, including, for instance, superconductivity at interfaces of non-superconducting copper oxides, ${ }^{1}$ the coexistence of superconductivity and ferromagnetism ${ }^{2,3}$ or orbital reconstruction. ${ }^{4}$ This is largely due to their structural, electronic and magnetic degrees of freedom, tuned by heteroepitaxial exposure and strain. ${ }^{5} \mathrm{~A}$ prominent example is the formation of a two-dimensional electron gas at the (001) interface between the two band insulators $\mathrm{SrTiO}_{3}$ (STO) and $\mathrm{LaAlO}_{3}(\mathrm{LAO}){ }^{6}$ Various ideas have been put forward to explain this, ${ }^{6-12}$ many of them related to the fact that ionic and heteropolar films of certain orientations consist of charged planes: this would lead to a sizeable potential along the film normal (polar catastrophe), unless its polar interfaces carry opposite compensating charge.

This charge can be provided by various reconstruction mechanisms, $6,7,10-15$ among them structural distortion effects and interface stoichiometry changes. A different possibility is electronic reconstruction, the pure transfer of charge between the opposite polar interfaces. ${ }^{6,7,14}$ It was originally suggested for the polar (111) surfaces of $\mathrm{K}_{3} \mathrm{C}_{60}$ (ref. 14) and later on for the (001) interface between the non-polar $\mathrm{SrTiO}_{3}$ and the polar $\mathrm{LaTiO}_{3}$ (ref. 7 ) or $\mathrm{LaMnO}_{3}{ }^{16}$ Apart from its conceptual appeal, electronic reconstruction might also form the basis for other interface effects such as superconductivity in LAO/STO (refs 2,3) and $\mathrm{YBa}_{2} \mathrm{Cu}_{3} \mathrm{O}_{6} /$ STO, ${ }^{17}$ or ferromagnetism in $\mathrm{LaMnO}_{3} / \mathrm{STO}$ and thin $\mathrm{LaCoO}_{3}$ films. ${ }^{18}$

Recent research has focused on the LAO/STO system, in which the conjectured electronic reconstruction would entail the transfer of $0.5 e^{-}$per two-dimensional unit cell (u.c.) from the LAO surface to the interface, where it would lead to a Ti valence reduction and to the observed two-dimensional electron gas in the intrinsically non-polar STO. LAO/STO and related TMO systems have been extensively studied both theoretically ${ }^{7,10}$ and by various experimental techniques, ${ }^{19-23}$ yielding an accordingly large body of data, which has provided valuable insight into the physics of polar TMO interfaces. $2,3,8,9,11,12,15,24-26$ However, different sample preparation and treatment ${ }^{2,6,8,9,24-26}$ often result in different oxygen stoichiometries. . $^{8,27,28}$ In addition, the variety of the used techniques, probes distinct physical quantities with different depth resolutions. Therefore, the reported LAO/STO interfacial electron concentrations vary widely and often deviate by orders of magnitude from values consistent with electronic reconstruction. $8,9,27-30$

Therefore, the origin of the two-dimensional electron gas in LAO/STO remains highly debated, and microscopic evidence is required, which supports the whole concept of electronic reconstruction in polar TMO films in general. Here we present such evidence, which we have collected in a judiciously chosen sample system devoid of several of the complications encountered in LAO/STO, namely $\mathrm{LaCoO}_{3}$ (LCO) on a polar substrate, and by using resonant $\mathrm{X}$-ray reflectivity (RXR), an experimental technique with sub-nanometre resolution and interface sensitivity.

\section{RESULTS}

Choice of the experimental technique

Various experimental techniques are being routinely used to explore the electronic properties of TMO heterostructures and interfaces, among them transport and Hall measurements, X-ray absorption (XAS), ${ }_{1}^{19,31}$ hard X-ray photoemission spectroscopy, ${ }^{20}$ resonant inelastic X-ray scattering, ${ }^{22}$ standing-wave excited $\mathrm{PES}^{23}$ or electron energy loss spectroscopy in scanning transmissionelectron geometry. ${ }^{21}$ These techniques exhibit different depth resolutions, mostly lacking interface sensitivity, and they probe different quantities such as the total charge density versus the

\footnotetext{
${ }^{1}$ Leibniz Institute for Solid State and Materials Research, IFW-Dresden, Dresden, Germany; ${ }^{2}$ Quantum Matter Institute, University of British Columbia, Vancouver, BC, Canada; ${ }^{3}$ Max Planck Institute for Solid State Research, Stuttgart, Germany; ${ }^{4}$ Materials Science and Technology Division, Oak Ridge National Laboratory, Oak Ridge, TN, USA; ${ }^{5}$ Department of Physics, Sungkyunkwan University, Suwon, Korea; ${ }^{6}$ Canadian Light Source, Saskatoon, SK, Canada; ${ }^{7}$ Department of Chemistry, University of British Columbia, Vancouver, BC, Canada; ${ }^{8}$ Max-Planck Institute for Chemical Physics of Solids, Dresden, Germany and ${ }^{9}$ Physikalisches Institut und Rokntgen Center for Complex Materials Systems (RCCM), Universität Würzburg, Am Hubland, Würzburg, Germany.

Correspondence: JE Hamann-Borrero (j.e.hamann.borrero@ifw-dresden.de) or V Hinkov (hinkov@physik.uni-wuerzburg.de)

Received 25 May 2016; revised 29 July 2016; accepted 5 August 2016
} 
a

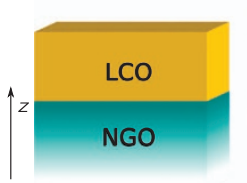

b

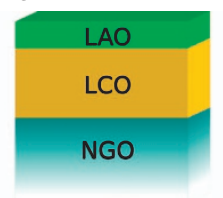

c

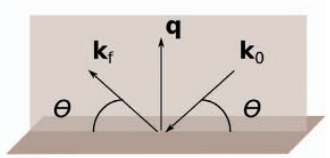

Figure 1. Schematic composition of the samples and scattering geometry. Both samples consist of $\mathrm{LaCoO}_{3}$ films, about 40 u.c. thick, grown by pulsed laser epitaxy on $\mathrm{NdO}$-terminated (001)- $\mathrm{NdGaO}_{3}$, with or without an additional LAO capping. All three materials are polar along the growth direction. We have chosen the $z$ axis of the coordinate system to point along the surface normal. (a) In the first sample, the polar-non-polar interface is vacuum/LCO. (b) In the second sample, the LCO surface is covered by two u.c. of LAO, and the polar-non-polar interface is shifted away from LCO to the LAO surface. (c) Specular scattering geometry with the transferred scattering wave vector $\mathbf{q}=\left(0,0, q_{z}\right)$ perpendicularly to the surface.

mobile charge density; this contributes to the uncertainty regarding the interfacial properties.

$\mathrm{RXR}^{4,32-38}$ is an element- and interface-specific technique, which can provide evidence for electronic reconstruction by directly probing the valence band electrons and the valence profile of the transition metal across the interface. It is non-destructive and does not alter the oxygen stoichiometry. Its full potential has not been exploited thus far due to difficulties in extracting real-space information from measured complex interference patterns. To overcome these difficulties we have developed a novel approach to RXR to determine valence depth profiles, which is described in later sections.

\section{Sample system}

There are several experimental complications associated with the vacuum/LAO/STO system: first, there is typically some degree of cation intermixing at the interface. Second, it contains two polarnon-polar (P/NP) interfaces. Finally, the polar LAO comprises elements with a rather stable, single valence, making electronic reconstruction within the LAO film itself prohibitively expensive. Therefore, we have chosen to study a $\mathrm{LaCoO}_{3}$ film ${ }^{39-42}$ on $\mathrm{NdGaO}_{3}$ (NGO) substrate. Both LCO and NGO are polar, and the $\mathrm{NdO}$ termination of $\mathrm{NGO}^{43}$ implies a LaO termination of the LCO film. Co is multivalent, and in LCO it is octahedrally coordinated with a nominal oxidation of $3+$. Our analysis below shows that reconstruction phenomena are confined to the film surface and the LCO//NGO interface remains unaltered, which simplifies the interpretation. Moreover, we were able to switch off the P/NP character of the vacuum/LCO interface in a controlled way by depositing a protective LAO layer on top: this moves the P/NP interface to the LAO surface and provides us with a second sample containing a reference polar-polar interface (Figure 1). The LCO system is not free from complications itself, which are associated with spin-state degrees of freedom, and which others ${ }^{18,40,41,44}$ and $w^{39,42}$ have studied extensively. However, these do not interfere with the valence-state study we present here.

The two samples-LCO//NGO and LAO/LCO//NGO-were grown using pulsed laser epitaxy and characterised as described in the Materials and Methods section. The total film thickness is about $15 \mathrm{~nm}$, including the 2 u.c. LAO capping in the LAO/LCO sample.

Obtaining the optical constants from XAS

XAS and RXR measurements were performed at $300 \mathrm{~K}$ with $\sigma$-polarised light, using the four-circle ultra-high vacuum diffractometer at the REIXS 10ID-2 beamline of the Canadian Light Source in Saskatoon, Canada (see Materials and Methods and ref. 45). The
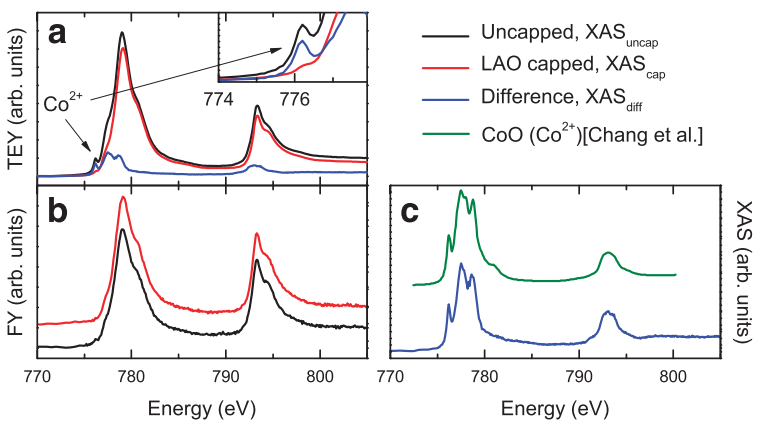

Figure 2. XAS spectra in the vicinity of the Co $L_{3}$ and $L_{2}$ absorption edges. Data for the uncapped sample are shown in black, and for the LAO-capped sample in red. (a) Spectra measured in TEY mode, scaled with respect to each other as described in the Materials and Methods section. The blue curve shows the difference spectrum, which we attribute to $\mathrm{Co}^{2+}$. The inset shows an enlargement around $776 \mathrm{eV}$, the energy at which $\mathrm{Co}^{2+}$ shows a characteristic prepeak. (b) Spectra measured in total-fluorescence yield (TFY) mode. (c) Comparison between our measured difference spectrum from (a), shown in blue, and results for $\mathrm{Co}^{2+}$ from ref. 46, shown in green.

XAS data of the two samples around the Co $L_{3}$ and $L_{2}$ absorption edges, measured in total electron yield (TEY) mode, which has a shallow probing depth of a few nanometres, are compared in Figure $2 \mathrm{a}$. The spectrum of the capped sample, $\mathrm{XAS}_{\text {cap }}$, represents stoichiometric $\mathrm{LaCo}^{3+} \mathrm{O}_{3}$ (high-spin- to low-spin-state ratio $\sim 1 / 2$, see refs 39,44$)$. The contribution from $\mathrm{Co}^{2+}$, which is observed in non-stoichiometric films, and which would result in a shoulder around $777.5 \mathrm{eV}$ and a prepeak around $776 \mathrm{eV}$, is negligible. In contrast, the spectrum of the uncapped sample, $\mathrm{XAS}_{\text {uncap, }}$ clearly shows non- $\mathrm{CO}^{3+}$ contributions with features indicative of $\mathrm{Co}^{2+}$. The fact that a capping of 2 u.c. is sufficient to suppress them, suggests that this Co with non-3+valence is localised near the surface. A further indication for that comes from a comparison with XAS measured in the bulk-averaging fluorescence yield mode in Figure $2 \mathrm{~b}$ : here the spectra of the capped and uncapped films are nearly indistinguishable and very much resemble the $\mathrm{Co}^{3+}$ spectrum shown in Figure 2a.

In order to assess the precise nature of the non- $\mathrm{Co}^{3+}$ contribution, we have subtracted the LAO/LCO//NGO spectrum from the LCO//NGO spectrum, scaling the spectra with respect to each other as described in the Materials and Methods section. In Figure $2 c$, we compare the difference spectrum $X S_{\text {diff }}$ with published experimental data of $\mathrm{Co}^{2+}$ in octahedral coordination. ${ }^{46}$ The agreement is excellent, strongly supporting the hypothesis of a structurally intact LCO surface containing $\mathrm{Co}^{2+}$.

\section{RXR measurements and fits}

In order to substantiate our findings we will next precisely determine the distribution of $\mathrm{Co}^{2+}$ by analysing RXR data, which were measured at and around the relevant absorption edges and which are shown in Figure 3. The novel analysis scheme we have developed to determine valence-state profiles is based on our approach in ref. 38 , and is described in the Materials and Methods section.

Here the properties to scrutinise are the surfacial and interfacial roughnesses, and the $\mathrm{Co}^{2+}$ concentration in a surface layer of variable thickness. Therefore, the initial layer sequence is $(\mathrm{O}$, organic contamination)/ $/ \mathrm{LaIO}_{3} / \mathrm{LaCo}^{3+} \mathrm{O}_{3} / \mathrm{NdGaO}_{3}$ for the $\mathrm{LAO} / \mathrm{LCO} / / \mathrm{NGO}$ sample and (O, organic contamination)/ $\mathrm{LaCO}_{1}^{2+}{ }_{x} \mathrm{Co}_{x}^{3+} \mathrm{O}_{3} / \mathrm{LaCo}^{3+} \mathrm{O}_{3} / \mathrm{NdGaO}_{3}$ for the LCO//NGO sample (Figure 4). We have included the first layer to account for oxygen adsorption to the surface ${ }^{47}$ and inevitable contaminations due to air exposure of the sample before the measurement. ${ }^{38}$ 
a

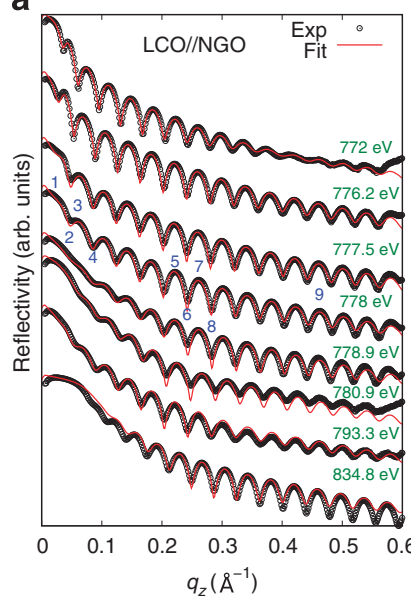

b

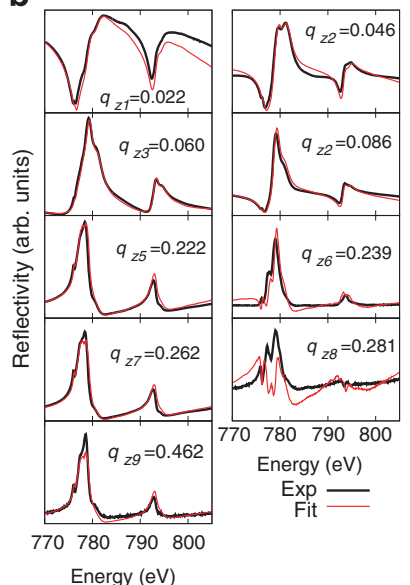

C

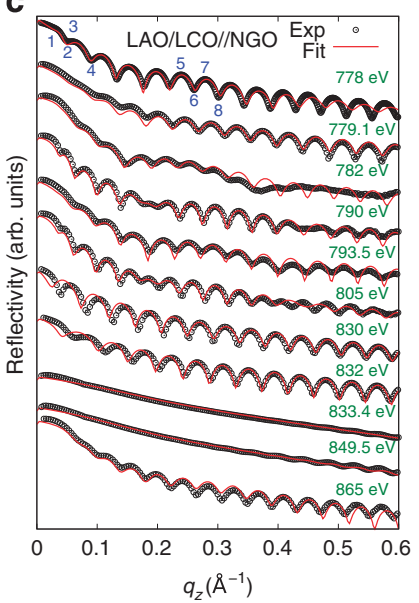

d

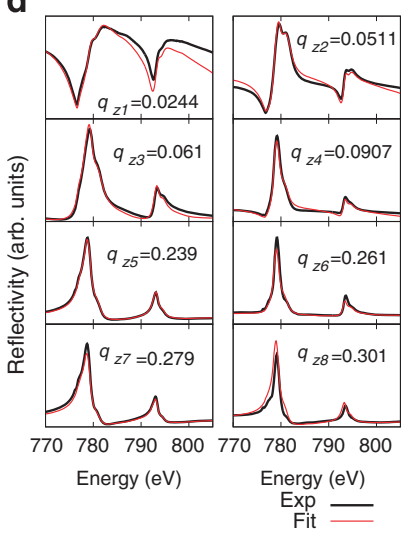

Figure 3. RXR data and fits. Data measured in the constant-energy and constant- $q_{z}$ modes (black symbols) are shown, along with the best obtained fits (red lines), based on the profiles shown in Figure 4. Data points at low $q_{z}$ were corrected for geometry effects ${ }^{54}$ (see also the Materials and Methods section). (a) Constant-energy scans for the uncapped sample. (b) Constant- $q_{z}$ scans for the uncapped sample. (c) Constant-energy scans for the capped sample. (d) Constant- $q_{z}$ scans for the capped sample. The constant-energy data are shown on a logarithmic scale, the constant- $q_{z}$ on a linear scale. For clarity, the scans have been shifted along the $y$ axis with respect to each other in ( $\mathbf{a}$ and $\mathbf{c}$ ). The constant $-q_{z}$ scans in ( $\mathbf{b}$ and $\mathbf{d}$ ) were measured at the $q_{z i}$ positions marked with blue numbers $i$ in (a and $\mathbf{c}$ ).

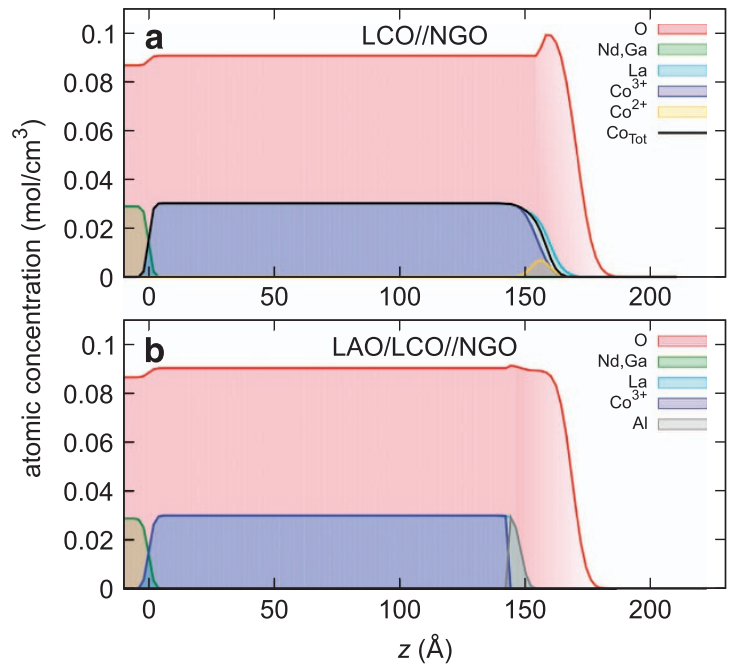

Figure 4. Element and valence depth concentration profiles. (a) Profiles of the uncapped sample. (b) Profiles of the capped sample. The region at the surface of the samples marked in lighter red is likely to contain further light elements such as carbon, in addition to oxygen. All results were obtained by the fitting procedure explained in the main text.

Details about how the implementation of the fitting procedure influences the quality of the fits are discussed in ref. 38 and references therein. In particular, the initial choice of a suitable layer sequence, and therefore of a reasonable number of parameters is crucial. If there are too many parameters, the convergence in a finite time is not guaranteed, and the fitting procedure might get trapped in local minima. For this reason, the substrate and the bulk of the films have been kept stoichiometric, a restriction that was not enforced at the surface to be able to track the individual cation profiles in detail. Also, all the elements have been allowed to converge towards individual roughnesses at the interfaces.

Starting with the initial layer sequence, we apply the fitting procedure described in the Materials and Methods and in ref. 38.
For both samples, we use constant-energy $\theta-2 \theta$ scans measured up to $q_{z}$-values of at least $0.58 \AA^{-1}$ and at various energies around the $\mathrm{La}$ and Co edges (Figure $3 \mathrm{a}, \mathrm{c}$ ), as well as constant- $q_{z}$ scans (Figure $3 \mathrm{~b}, \mathrm{~d}$ ) at the marked $q_{z}$-positions. Panels $\mathrm{a}$ and $\mathrm{b}$ also contain the final fits for the LAO/LCO//NGO sample, while panels $\mathrm{C}$ and $d$ contain those for the LCO//NGO sample. The corresponding final elemental and valence depth profiles are shown in Figure 4.

We will now elaborate on important details of the fit. An important property of the LAO/LCO//NGO RXR data, which our fit correctly reproduces, is the absence of $\mathrm{Co}^{2+}$-related structures around $777.5 \mathrm{eV}$ at any wave number $q_{z}$. This confirms that there is no $\mathrm{Co}^{2+}$ in this sample in appreciable amounts. In contrast, the RXR data of the uncapped LCO//NGO sample exhibit prominent features around $777.5 \mathrm{eV}$ at high $q_{z}$, a qualitative indication of a $\mathrm{Co}^{2+}$ accumulation localised in depth. Indeed, the quantitative fit reveals a narrow $\mathrm{Co}^{2+}$ distribution at the film surface with a full width at half maximum of $8.6 \AA$ ( 2 u.c.), concomitant with a decline in $\mathrm{Co}^{3+}$ concentration (Figure 4a). Importantly, the total amount of $\mathrm{Co}^{2+}$, if it was confined to one monolayer, would correspond to an areal density of $0.55 \pm 0.15$ ions per u.c., close to half coverage.

The fits demonstrate that both samples exhibit a sharp substrate-film interface and thus grow with very good quality (Figure 3). Our analysis also suggests that the La profile extends about half a u.c. further than the Co profile, which is consistent with a predominant $\mathrm{LaO}$ termination and is expected from the $\mathrm{NdO}$ termination of the substrate. This, and the fact that we achieve our fits using $\mathrm{Co}^{2+}$ and $\mathrm{Co}^{3+}$ spectra typical for bulk cobaltates, strongly indicate that both samples maintain their crystallinity up to the LCO surface, and are not subject to chemical decomposition.

We have confirmed the quality and robustness of our fits in several ways. First, the successful determination of chemical and valence-state profiles crucially depends on how different the optical constants of the involved species are. From Figure 2 it is clear that $\mathrm{Co}^{3+}$ and $\mathrm{Co}^{2+}$ have distinctly different resonant absorption edges. $\mathrm{Co}^{2+}$ can be particularly well identified by the prepeak at $777.5 \mathrm{eV}$, and we have exploited this to distinguish the profiles of the two Co valences. The fact that the signatures of $\mathrm{Co}^{2+}$ are stronger at the Kiessig fringe minima (Figure $3 \mathrm{~b}$, right column) than at the maxima (left column), and that the fits at the 
a

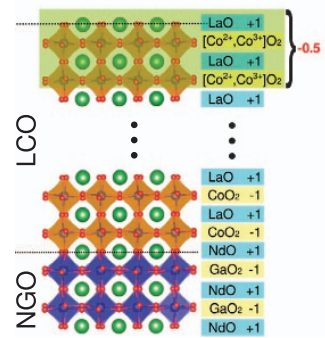

b

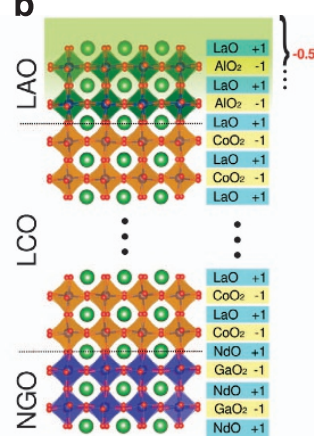

Figure 5. Crystal structures and schematic charge and valence profiles for both samples. (a) Uncapped sample with the electronically reconstructed surface following from our analysis. (b) Sample capped with LAO. The reconstruction of the LAO surface is not known and beyond the scope of this work. A charge of $-0.5 e$ proximate to the surface follows from the reconstruction.

maxima are superior to those at the minima fully support our conclusion, namely that $\mathrm{Co}^{2+}$ is limited to the surface: the maxima reflect the superposition of surface and interface signal, and are therefore dominated by $\mathrm{Co}^{3+}$. The minima, on the other hand, reflect the difference between surface $\left(\mathrm{Co}^{2+}\right.$ and $\left.\mathrm{Co}^{3+}\right)$ and substrate interface signal $\left(\mathrm{Co}^{3+}\right.$ only), and are therefore particularly sensitive to $\mathrm{Co}^{2+}$. In addition, the minima are much sharper than the maxima and thus more prone to even small deviations in the elemental profiles.

Second, altering the $\mathrm{Co}^{2+}$ concentration from the 0.55 ions per u.c. quickly deteriorates the fit quality $\left(x^{2}\right)$ and distorts the signatures of $\mathrm{Co}^{2+}$ both at minima and maxima. We use this sensitivity to estimate an error bar of 0.15 ions per u.c., which indicates the range of $\mathrm{Co}^{2+}$ concentrations within which the $x^{2}$ of the fit deviates by $<15 \%$ from the best fit. Also, it is qualitatively observed that for concentrations outside this range the $\mathrm{Co}^{2+}$ features in the RXR simulations strongly under- or overestimate the corresponding features in the measured RXR data. This also demonstrates that the total $\mathrm{Co}^{2+}$ concentration, which characterises the electronic system, is a sensitive fitting parameter, as can be also expected on theoretical grounds (cf. references in ref. 38).

Third, further simulations showed that there is no accumulation of $\mathrm{Co}^{2+}$ near the LCO//NGO interface in either of the two samples. This indicates that LCO and NGO are of comparable polarity and the interface is not involved in the reconstruction.

Finally, we have tried to use Co optical constants based on XAS data from the LCO//NGO sample only, without subtracting the $\mathrm{LAO} / \mathrm{LCO} / \mathrm{NGO}$ spectrum. Fitting the Co profile in LCO//NGO reflectivity data with these optical constants did not result in a satisfactory fit, confirming that the film does not consist of a homogeneous mixture of $\mathrm{Co}^{2+}$ and $\mathrm{Co}^{3+}$.

\section{DISCUSSION}

How can we interpret the valence-state reconstruction we observe in LCO? Electrostatic considerations dictate that a polar sample of the given thickness must reconstruct in some manner. ${ }^{13-15}$ The following facts and findings we have established in the previous section are important when addressing the associated mechanism: first, the chemical integrity of the sample surface; second, the $\mathrm{NdO}$ termination of the substrate, which implies an LaO termination of the film; and third, the fact that the amount of charge necessary to induce the observed $\mathrm{Co}^{2+}$ is close to $-0.5 e$ u.c.

The reconstruction scenario, applied to the LCO surface, is outlined in Figures 5 and 6 . Beginning from the surface, the atomic layer sequence is $\mathrm{LaO} / \mathrm{CoO}_{2} / \mathrm{LaO} / \mathrm{CoO}_{2} / \ldots$, which nominally
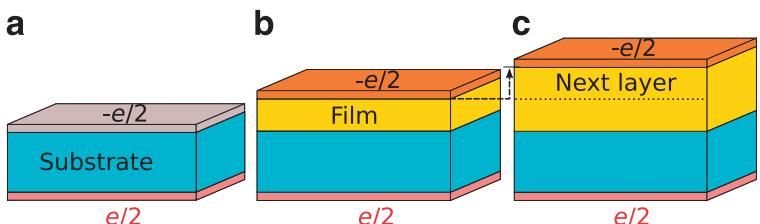

Figure 6. Reconstruction scheme at different stages during the epitaxial sample growth. (a) The reconstructed substrate surface and backside carry effective charges of $-e / 2$ and $e / 2$ per u.c., respectively. (b,c) With each newly deposited film monolayer, the negative charge travels to the sample surface, whereas the charge at the substrate back remains unchanged.

corresponds to a charge concentration sequence of $+e /-e /+e /$ $-e / . .$. per u.c. over the sample thickness, including the entire substrate (Figure 5). The compensation of the associated internal potential occurs successively during epitaxial growth: the NdOterminated, polar substrate has undergone a reconstruction by an unidentified mechanism, resulting in effective charges of $-e / 2$ and $e / 2$ per u.c. at its surface and backside, respectively (Figure 6a). Upon successively depositing LCO monolayers, the surface reconstruction heals and the effective negative charge is established at the new film surface (Figure $6 b, c)$, thereby keeping the internal potential in the now thicker sample compensated.

$\mathrm{La}$ and $\mathrm{O}$ have a rather stable single valence and do not exhibit bands near the chemical potential, whereas calculations, for instance within the framework of $L D A+U$, have established that the first electron affinity state is within a Co $d$-band. ${ }^{48}$ Therefore, it is energetically favourable to leave the potential uncompensated over the topmost half u.c. and accommodate the compensating charge in the buried $\mathrm{CoO}_{2}$ layer, leading to the reconstructed surface configuration $\mathrm{LaO}(+1) / \mathrm{CoO}_{2}(-1.5)$. This changes the $\mathrm{Co}$ valence to $2.5+$, which we spectroscopically observe as a superposition of $\mathrm{Co}^{3+}$ and $\mathrm{Co}^{2+}$ (Figure 2). This electronic reconstruction scenario involving the subsurface layer is very different from the scenarios discussed for LAO/STO and related materials.

We will next consider different reconstruction scenarios. On the one hand, whereas chemical reconstruction or the surface deposition of anions such as $\mathrm{OH}^{-}$could hypothetically prevent the polar catastrophe, these mechanisms do not involve the creation of $\mathrm{Co}^{2+}$. On the other hand, the deposition of electropositive elements, e.g., in an additional LaO layer, or the creation of oxygen vacancies could explain the presence of $\mathrm{Co}^{2+}$, but would not prevent the polar catastrophe-therefore, the energy costs associated with such disruptions are not justified. These arguments, the close proximity of the observed $\mathrm{Co}^{2+}$ concentration to 0.5 per u.c. and the fact that it is energetically favourable to change the Co valence in a purely electronical way ${ }^{48}$ leave electronic reconstruction as the explanation by far most consistent with our data.

Importantly, based on XAS alone only very qualitative information can be obtained about the elemental and valence depth distribution by taking advantage of the different probing depth of the TEY and fluorescence yield mode extraction (see above). Our investigations indicate that even bulk properties should be better studied by RXR: surface reconstructions, stoichiometry variations and contaminations are ubiquitous effects. Using TEY, which typically has a probing depth of a few nanometres, will grossly overemphasise surface effects. As a case in point consider our TEY results: if interpreted as being representative of bulk properties, they would falsely indicate a bulk $\mathrm{Co}^{2+} / \mathrm{Co}^{3+}$ ratio of $1 / 4$, whereas the actual bulk $\mathrm{Co}^{2+}$ content is negligible.

Using the more bulk sensitive fluorescence yield mode is not a viable alternative either, as it does not strictly represent absorption. ${ }^{49,50}$ The best option whether RXR is not available is to terminate the sample with a protective layer. However, in many 
cases it will be difficult to find a material that does not itself change the film properties: it has to be lattice matched, of the same polarity, of comparable oxygen affinity and so on.

In summary, we have demonstrated that RXR is an excellent tool to study reconstruction phenomena in heterostructures comprising complex materials. We have shown direct microscopic evidence for electronic reconstruction on the polar (001) surface of $\mathrm{LaCoO}_{3}$. Whereas a RXR analysis of the magnetic structure of LCO has to await future studies, our results indicate that LCO films are dominated by $\mathrm{Co}^{3+}$ and that $\mathrm{Co}^{2+}$ is limited to the surface: this sets stringent boundary conditions for the interpretation of ferromagnetism in LCO thin films ${ }^{42,44}$ and powders. ${ }^{18}$ It suggests that $\mathrm{Co}^{3+}$ spin-state transitions in the bulk drive the ferromagnetism and excludes schemes involving the presence of $\mathrm{Co}^{4+}$ at the surface. ${ }^{18}$

Our results stress the necessity to further develop RXR into a tool to routinely extract optical constants of the bulk and of buried interfaces. This is not only important in the presence of surface reconstruction but also when the detection of TEY drain current is technically difficult or not possible, such as in insulators, in the presence of strong magnetic fields and in femtosecond-resolved pump-probe experiments. Corresponding experimental endeavours are underway and our approach is ideally suited to analyse the resulting data.

\section{MATERIALS AND METHODS}

Sample synthesis

We used pulsed laser epitaxy to grow LCO thin films with (001) orientation on NdO-terminated single-crystal NGO substrates with (001) orientation (pseudocubic notation). A KrF excimer laser $(\lambda=248 \mathrm{~nm})$ with a laser fluence of $\sim 1 \mathrm{~J} / \mathrm{cm}^{2}$ was used for ablating sintered LCO and single-crystal LAO targets. The samples were fabricated at $700^{\circ} \mathrm{C}$ in 100 mTorr of oxygen partial pressure. We note that the growth at high oxygen partial pressure was necessary for high-quality, phase-pure LCO thin films. Lower oxygen partial pressure resulted in cobalt oxide impurity phases as evidenced by X-ray diffraction.

\section{Optical constant determination by XAS}

The off-resonant, imaginary parts of the atomic scattering factors of the involved elements, as well as those parts lying outside of the scrutinised XAS energy window of $\sim 600-900 \mathrm{eV}$ were retrieved from Chantler tables. ${ }^{51}$ The resonant parts of $\mathrm{Co}^{3+}$ and La were obtained from XAS measurements in TEY mode. The background was corrected and the data aligned with the tabulated values as previously described in ref. 4 to obtain the complete imaginary parts of the atomic scattering factors. The real parts were obtained by Kramers-Kronig transformation.

For the $\mathrm{Co}^{2+}$, intermediate steps were necessary: as described in the main text, a $\mathrm{Co}^{3+}$ spectrum was subtracted from the data of the uncapped $\mathrm{LCO} / / \mathrm{NGO}$ sample (Figure 2). The difference spectrum $\mathrm{XAS}_{\text {diff }}$ depends on the scaling of the original spectra with respect to each other. However, the choice of the scaling underlies stringent restrictions: $X_{\text {diff }}$ may not become negative at any energy, it must reflect the edge jump ${ }^{51}$ and it should represent a meaningful Co valence. These restrictions lead to a close to perfect $\mathrm{Co}^{2+}$ spectrum (Figure 2) in octahedral high-spin configuration. Different scaling choices do not lead to either of the other possible Co valencies ( $3+$ and $4+$ ) in either of the known spin states, or mixtures thereof. To further improve the alignment of the cobalt spectra to the Chantler calculations, which is somewhat complicated by the vicinity of the La resonance, we have compared the areas below the $\mathrm{Co}^{3+}$ and the $\mathrm{Co}^{2+}$ resonances. For a purely ionic situation, the ratio is $4 / 3$. We expect covalence effects, which are significantly stronger for $\mathrm{Co}^{3+}$ than for $\mathrm{Co}^{2+}$, to reduce this ratio to $\sim 1.1$. In the aligning process of the two spectra to the Chantler calculations, we have ensured that this ratio be kept.

\section{Valence-state profiling based on RXR data}

Our approach here is based on the chemical profiling scheme we describe in ref. 38. The sequence of steps to perform is as follows: first, we set up a model consisting of a sequence of layers $k$ containing elements (in their respective valence) $i$, with the parameters being the thickness $t_{k}$, atomic concentration $c_{i, k}$ and roughness of the respective upper interface $\sigma_{i, k}$. We then simulate the resulting RXR curves, compare them with the measured curves and optimise the parameters until convergence is achieved. The initial layer sequence depends on the complexity of the system and reflects preliminary information about surfactant layers, intermixing regions and so on. For the samples studied here, it is discussed in the main text.

From the parameters of the individual layers $t_{k}, c_{i, k}$ and $\sigma_{i, k}$, we obtain the elemental depth concentration profiles $c_{i}(z)$ of the entire heterostructure, such as those shown in Figure 4. Their properties are described by atomic scattering factors $f_{i}(\omega)$ with a unique photon frequency dependence, which we derive from the XAS spectra as described above., ${ }^{4,38}$ The entire heterostructure is then described by the depth-resolved susceptibility

$$
\chi(z, \omega)=\frac{4 \pi}{\left|\mathbf{k}_{0}\right|^{2}} r_{\mathrm{el}} \sum_{i} N_{\mathrm{A}} c_{i}(z) f_{i}(\omega)
$$

where $\mathbf{k}_{0}$ is the wave vector of the incoming beam, $r_{\mathrm{el}}$ is the classical electron radius and $N_{A}$ is the Avogadro number. On the basis of $\chi(z, \omega), \operatorname{RXR}$ spectra can be simulated using the Parratt formalism. ${ }^{52}$ Finally, a leastsquares algorithm is used to optimise $x^{2}$, the deviation between the measured data and the simulated spectra. ${ }^{38,53}$

At low $q_{z}$-values (below about $0.05 \AA^{-1}$ ) the measured intensity is suppressed due to geometrical shadowing effects and deviates from the expected intensity. We have applied a corresponding correction factor. ${ }^{54}$

\section{ACKNOWLEDGEMENTS}

We thank B Keimer and K Fürsich for helpful discussions. This work has been supported by the German Research Foundation DFG under grant number HA6470/1-2 and through SFB 1170 'ToCoTronics' (project C04), as well as by the Canadian organisations NSERC, CFI and CRC. WSC was supported by the Basic Science Research Program through the National Research Foundation of Korea (NRF) funded by the Ministry of Science, ICT and future Planning (NRF-2014R1A2A2A01006478). Parts of the research were performed at the Max-Planck UBC Centre for Quantum Materials. Parts of the research were performed at the Canadian Light Source, which is funded by the Canada Foundation for Innovation, the Natural Sciences and Engineering Research Council of Canada, the National Research Council Canada, the Canadian Institutes of Health Research, the Government of Saskatchewan, Western Economic Diversification Canada and the University of Saskatchewan. The work at ORNL was supported by the U.S. Department of Energy, Office of Science, Basic Energy Sciences, Materials Sciences and Engineering Division.

\section{CONTRIBUTIONS}

W.S.C. and H.N.L. synthesised and characterised the samples; J.E.H.-B., R.S., F.H. and A.R. performed the measurements; SM wrote the R.X.R. analysis programme; J.E.H.-B., S.M., R.J.G., V.B.Z. and V.H. analysed the data; J.E.H.-B., I.E., R.J.G., MWH, V.B.Z., G.A.S. and V.H. interpreted the results with support from all authors; J.E.H.-B. and V.H. wrote the manuscript, on which all authors commented and agreed; V.H. coordinated the project.

\section{COMPETING INTERESTS}

The authors declare no conflict of interest.

\section{REFERENCES}

1. Gozar, A. et al. High-temperature interface superconductivity between metallic and insulating copper oxides. Nature 455, 782 (2008).

2. Li, L., Richter, C., Mannhart, J. \& Ashoori, R. C. Coexistence of magnetic order and two-dimensional superconductivity at $\mathrm{LaAlO}_{3} / \mathrm{SrTiO}_{3}$ interfaces. Nat. Phys. 7, 762-766 (2011).

3. Bert, J. A. et al. Direct imaging of the coexistence of ferromagnetism and superconductivity at the $\mathrm{LaAlO}_{3} / \mathrm{SrTiO}_{3}$ interface. Nat. Phys. 7, 767-771 (2011).

4. Benckiser, E. et al. Orbital reflectometry of oxide heterostructures. Nat. Mater. 10, 189-193 (2011).

5. Hwang, H. Y. et al. Emergent phenomena at oxide interfaces. Nat. Mater. 11, 103 (2012).

6. Ohtomo, A. \& Hwang, H. Y. A high-mobility electron gas at the $\mathrm{LaAlO}_{3} / \mathrm{SrTiO}_{3}$ heterointerface. Nature 427, 423 (2004).

7. Okamoto, S. \& Millis, A. J. Electronic reconstruction at an interface between a mott insulator and a band insulator. Nature 428, 630-633 (2004).

8. Kalabukhov, A. et al. Effect of oxygen vacancies in the $\mathrm{SrTiO}_{3}$ substrate on the electrical properties of the $\mathrm{LaAlO}_{3} / \mathrm{SrTiO}_{3}$ interface. Phys. Rev. B 75, 121404 (2007). 
9. Siemons, W. et al. Origin of charge density at $\mathrm{LaAlO}_{3}$ on $\mathrm{SrTiO}_{3}$ heterointerfaces: possibility of intrinsic doping. Phys. Rev. Lett. 98, 196802 (2007).

10. Pentcheva, R. \& Pickett, W. E. Avoiding the polarization catastrophe in $\mathrm{LaAlO}_{3}$ overlayers on $\mathrm{SrTiO}_{3}(001)$ through polar distortion. Phys. Rev. Lett. 102, 107602 (2009).

11. Jia, C. L. et al. Oxygen octahedron reconstruction in the $\mathrm{SrTiO}_{3} / \mathrm{LaAlO}_{3}$ heterointerfaces investigated using aberration-corrected ultrahigh-resolution transmission electron microscopy. Phys. Rev. B 79, 081405 (2009).

12. Liu, Z. Q. et al. Origin of the two-dimensional electron gas at $\mathrm{LaAlO}_{3} / \mathrm{SrTiO}_{3}$ interfaces: the role of oxygen vacancies and electronic reconstruction. Phys. Rev. $X$ 3, 021010 (2013).

13. Harrison, W. A., Kraut, E. A., Waldrop, J. R. \& Grant, R. W. Polar heterojunction interfaces. Phys. Rev. B 18, 4402-4410 (1978).

14. Hesper, R., Tjeng, L. H., Heeres, A. \& Sawatzky, G. A. Photoemission evidence of electronic stabilization of polar surfaces in $\mathrm{K}_{3} \mathrm{C}_{60}$. Phys. Rev. B 62, 16046-16055 (2000).

15. Noguera, C. Polar oxide surfaces. J. Phys.: Condens. Matter 12, R367 (2000).

16. Wang, X. R. et al. Imaging and control of ferromagnetism in $\mathrm{LaMnO}_{3} / \mathrm{SrTiO}_{3}$ heterostructures. Science 349, 716-719 (2015).

17. Pavlenko, N., Elfimov, I., Kopp, T. \& Sawatzky, G. A. Interface hole doping in cuprate-titanate superlattices. Phys. Rev. B 75, 140512 (2007).

18. Yan, J.-Q., Zhou, J.-S. \& Goodenough, J. B. Ferromagnetism in $\mathrm{LaCoO}_{3}$. Phys. Rev. $B$ 70, 014402 (2004).

19. Stöhr, J. \& Siegmann, H. C. Magnetism-From Fundamentals to Nanoscale Dynamics. (Springer, 2006).

20. Rubio-Zuazo, J. \& Castro, G. Non-destructive compositional depth profile analysis by hard X-ray photoelectron spectroscopy. J. Phys. Conf. Ser. 100, 012042 (2008).

21. Muller, D. A. Structure and bonding at the atomic scale by scanning transmission electron microscopy. Nat. Mater. 8, 263-270 (2009).

22. Berner, G. et al. $\mathrm{LaAlO}_{3} / \mathrm{SrTiO}_{3}$ oxide heterostructures studied by resonant inelastic X-ray scattering. Phys. Rev. B 82, 241405 (2010).

23. Gray, A. X. et al. Interface properties of magnetic tunnel junction $\mathrm{La}_{0.7} \mathrm{Sr}_{0.3} \mathrm{MnO}_{3} /$ $\mathrm{SrTiO}_{3}$ superlattices studied by standing-wave excited photoemission spectroscopy. Phys. Rev. B 82, 205116 (2010).

24. Thiel, S., Hammerl, G., Schmehl, A., Schneider, C. W. \& Mannhart, J. Tunable quasi-twodimensional electron gases in oxide heterostructures. Science 313, 1942-1945 (2006).

25. Brinkman, A. et al. Magnetic effects at the interface between nonmagnetic oxides. Nat. Mater. 6, 493-496 (2007).

26. Warusawithana, M. P. et al. $\mathrm{LaAlO}_{3}$ stoichiometry is key to electron liquid formation at $\mathrm{LaAlO}_{3} / \mathrm{SrTiO}_{3}$ interfaces. Nat. Commun. 4, 2351 (2013).

27. Herranz, G. et al. High mobility in $\mathrm{LaAlO}_{3} / \mathrm{SrTiO}_{3}$ heterostructures: origin, dimensionality, and perspectives. Phys. Rev. Lett. 98, 216803 (2007).

28. Basletic, M. et al. Mapping the spatial distribution of charge carriers in $\mathrm{LaAlO}_{3} / \mathrm{SrTiO}_{3}$ heterostructures. Nat. Mater. 7, 621 (2008).

29. Nakagawa, N., Hwang, H. Y. \& Muller, D. A. Why some interfaces cannot be sharp. Nat. Mater. 5, 204-209 (2006).

30. Sing, M. et al. Profiling the interface electron gas of $\mathrm{LaAlO}_{3} / \mathrm{SrTiO}_{3}$ heterostructures with hard X-ray photoelectron spectroscopy. Phys. Rev. Lett. 102, 176805 (2009).

31. de Groot, F. \& Kotani, A. Core Level Spectroscopy of Solids. (CRC Press, 2008).

32. Kao, $C$. et al. Magnetic-resonance exchange scattering at the iron $L_{\| 1}$ and $L_{\|||}$edges. Phys. Rev. Lett. 65, 373-376 (1990).

33. Kim, D. et al. Chemical depth profile of passive oxide on stainless steel. Appl. Phys. Lett. 85, 6427-6429 (2004).

34. Smadici, S. et al. Electronic reconstruction at $\mathrm{SrMnO}_{3}-\mathrm{LaMnO}_{3}$ superlattice interfaces. Phys. Rev. Lett. 99, 196404 (2007).

35. Tonnerre, J. M. et al. Depth magnetization profile of a perpendicular exchange coupled system by soft-X-ray resonant magnetic reflectivity. Phys. Rev. Lett. 100, 157202 (2008).

36. Als-Nielsen, J. \& McMorrow, D. Elements of Modern X-ray Physics. (Wiley, 2011).

37. Fink, J., Schierle, E., Weschke, E. \& Geck, J. Resonant elastic soft X-ray scattering. Rep. Prog. Phys. 76, 056502 (2013).

38. Macke, S. et al. Element specific monolayer depth profiling. Adv. Mater. 26, 6554 (2014).

39. Haverkort, M. W. et al. Spin state transition in $\mathrm{LaCoO}_{3}$ studied using soft X-ray absorption spectroscopy and magnetic circular dichroism. Phys. Rev. Lett. 97, 176405 (2006)

40. Freeland, J. W., Ma, J. X. \& Shi, J. Ferromagnetic spin-correlations in strained $\mathrm{LaCoO}_{3}$ thin films. Appl. Phys. Lett. 93, 212501 (2008).

41. Merz, M. et al. X-ray absorption and magnetic circular dichroism of $\mathrm{LaCoO}_{3}$, $\mathrm{La}_{0.7} \mathrm{Ce}_{0.3} \mathrm{CoO}_{3}$, and $\mathrm{La}_{0.7} \mathrm{Sr}_{0.3} \mathrm{CoO}_{3}$ films: evidence for cobalt-valence-dependent magnetism. Phys. Rev. B 82, 174416 (2010).

42. Choi, W. S. et al. Strain-induced spin states in atomically ordered cobaltites. Nano Lett. 12, 4966-4970 (2012).

43. Ohnishi, T. et al. A-site layer terminated perovskite substrate: $\mathrm{NdGaO}_{3}$. Appl. Phys. Lett. 74, 2531-2533 (1999).

44. Pinta, C. et al. Suppression of spin-state transition in epitaxially strained lacoo ${ }_{3}$. Phys. Rev. B 78, 174402 (2008).

45. Hawthorn, D. G. et al. An in-vacuum diffractometer for resonant elastic soft X-ray scattering. Rev. Sci. Instrum. 82, 073104 (2011).

46. Chang, C. F. et al. Spin blockade, orbital occupation, and charge ordering in $\mathrm{La}_{1.5} \mathrm{Sr}_{0.5} \mathrm{CoO}_{4}$. Phys. Rev. Lett. 102, 116401 (2009).

47. Kushima, A., Yip, S. \& Yildiz, B. Competing strain effects in reactivity of $\mathrm{LaCoO}_{3}$ with oxygen. Phys. Rev. B 82, 115435 (2010).

48. Korotin, M. A. et al. Intermediate-spin state and properties of $\mathrm{LaCoO}_{3}$. Phys. Rev. $B$ 54, 5309-5316 (1996).

49. Kurian, R. et al. Intrinsic deviations in fluorescence yield detected X-ray absorption spectroscopy: the case of the transition metal $L_{2,3}$ edges. J. Phys.: Condens. Matter 24, 452201 (2012).

50. Green, R. J. et al. Comment on 'state-dependent electron delocalization dynamics at the solute-solvent interface: soft-X-ray absorption spectroscopy and ab initio calculations'. Phys. Rev. Lett. 112, 129301 (2014).

51. Chantler, C. T. Theoretical form factor, attenuation, and scattering tabulation for $\mathrm{Z}=1-92$ from $\mathrm{E}=1-10 \mathrm{eV}$ to $\mathrm{E}=0.4-1.0 \mathrm{MeV}$. J. Phys. Chem. Ref. Data 24, 71-643 (1995).

52. Parratt, L. G. Surface studies of solids by total reflection of X-rays. Phys. Rev. $\mathbf{9 5}$, 359-369 (1954).

53. Press, W., Flannery, B., Teukolsky, S., Vetterling, W. et al. Numerical Recipes vol. 547. Cambridge Univ. Press, (1986).

54. Gibaud, A., Vignaud, G. \& Sinha, S. K. The correction of geometrical factors in the analysis of X-ray reflectivity. Acta Crystallogr. A Found. Crystallogr. 49, 642-648 (1993).

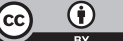
article are included in the article's Creative Commons license, unless indicated otherwise in the credit line; if the material is not included under the Creative Commons license, users will need to obtain permission from the license holder to reproduce the material. To view a copy of this license, visit http://creativecommons.org/licenses/ by/4.0/

(c) The Author(s) 2016 